\title{
O impacto da fisioterapia na funcionalidade após ressecção tumoral através da sacrectomia parcial associado à técnica de criocongelamento: relato de caso
}

\author{
The impact of physical therapy on functionality after tumor resonance through sacrectomy \\ partially associated with criocogelament technique: case report
}

\author{
José Carlos Alves Fabrício Júnior ${ }^{1}$, Andréia Basta ${ }^{2}$, Eduardo Yonamine Sadao ${ }^{3}$, Karen Voltan ${ }^{4}$, \\ Letícia Correia Monte ${ }^{1}$, Nilza Aparecida Almeida de Carvalho ${ }^{6}$
}

\section{Resumo}

Introdução: Tumores ósseos malignos consistem aproximadamente $7 \%$ das neoplasias primárias em indivíduos menores de 20 anos de idade. Dentre todos esses, a maior prevalência é do Osteossarcoma, ocorrendo em 8,7 casos/miIhão e seguido pelo Sarcoma de Ewing com 2,9 casos/milhão. Objetivo: Descrever o efeito da fisioterapia na funcionalidade do paciente submetido à ressecção tumoral através da sacrectomia parcial associado ao Criocongelamento. Relato de Caso: Paciente do sexo feminino LCM de 26 anos com queixa de dor lombar a 2 anos e um abaulamento na região do sacro, apresentou imagem de ressonância magnética sugestiva de neoplasia na região anterior ao Sacro. Após investigação com grupo do Tumor da IMSC-SP e através da biópsia foi diagnosticado um cordoma de Sacro. No dia

1. Fisioterapeuta Especialista pela Irmandade da Santa Casa de São Paulo. Especialização em Fisioterapia Músculo Esquelética. São Paulo-SP - Brasil

2. Professora e Supervisora da Irmandade da Santa Casa de São Paulo. Curso de Especialização em Fisioterapia Músculo Esquelética. São Paulo-SP - Brasil

3. Professor Adjunto da Faculdade de Ciências Médicas da Santa Casa de São Paulo. Departamento de Ortopedia e Traumatologia. Chefe do Grupo de Oncologia Ortopédica da Irmandade da Santa Casa de Misericórdia de São Paulo. São Paulo - SP - Brasil

4. Médica Assistente da Irmandade da Santa Casa de Misericórdia de São Paulo. Grupo de Oncologia Ortopédica. São Paulo - SP Brasil

5. Fisioterapeuta Chefe do Curso de Fisioterapia Musculoesquelética da Irmandade da Santa Casa de Misericórdia de São Paulo. São Paulo-SP - Brasil

Trabalho realizado: Irmandade da Santa Casa de Misericórdia de São Paulo. Setor de Fisioterapia. São Paulo-SP - Brasil

Endereço para correspondência: José Carlos Alves Fabrício Júnior. Irmandade da Santa Casa de Misericórdia de São Paulo. Setor de Fisioterapia. Rua Dr. Cesário Mota Junior, 112 - Vila Buarque - 01221-020 - São Paulo - SP - Brasil. E-mail:fabriciojr_fisio@hotmail.com
17 de maio foi realizada a ressecção da lesão através de uma sacrectomia parcial associado à técnica de criocongelamento. Após a cirurgia a paciente recebeu acompanhamento com a equipe de fisioterapia realizando uma avaliação funcional através do Escore Musculoskeletal Tumor Society (MSTS) e o Toronto Extremity Salvage Score - TESS. Resultados: De acordo com escore MSTS após um ano de tratamento a paciente apresentou Pré-tratamento (Ruim), Pós-Tratamento (Bom), o escore TESS paciente melhorou de $28 \%$ para $48 \%$. Conclusão: Oacompanhamento multidisciplinar do grupo do Tumor e a equipe de fisioterapia se mostrou eficaz garantindo em 1 ano de tratamento uma melhora funcional de acordo com os escores aplicados.

Descritores: Oncologia, Sacro/cirurgia, Cordoma, Modalidade de fisioterapia, Crioterapia/métodos, Criocirurgial métodos
Abstract
Introduction: Malignant bone tumors comprise approximately $7 \%$ of primary neoplasms in patients under 20 years of age. Among all these, the highest prevalence is in osteosarcoma, occurring in 8.7 cases / million, followed by Ewing's sarcoma with 2.9 casos/milhão. Objective: To describe the intervention of Physical Therapy after criocongelamento Sacro. Case Report: A female patient LCM of 26 years complaining of back pain at 2 years and a bulging in the sacral region, magnetic resonance imaging showed suggestive of neoplasia in the region prior to the Sacro. After investigation with the group Tumor IMSC-SP and through biopsy was diagnosed with chordoma of Sacro. On the 17th of May was performed resection of the lesion through a partial sacrectomia associated with criocongelamento technique. After surgery the patient was monitored with the physiotherapy team performing a functional assessment score through the Musculoskeletal Tumor Society (MSTS) and Toronto Extremity Salvage Score-TESS. Results: According to MSTS score after one 
Fabrício Júnior JCA, Basta A, Sadao EY, Voltan K, Monte LC, Carvalho NAA. O impacto da fisioterapia na funcionalidade após ressecção tumoral através da sacrectomia parcial associado à técnica de criocongelamento: relato de caso.

year of treatment the patient had pretreatment (Bad), PostTreatment (good), the TESS score patient improved from $28 \%$ to $48 \%$. Conclusion: The multidisciplinary group of Tumor and physiotherapy team was effective providing at 1 year of treatment one functional improvement according scores applied.

Keywords: Medical oncology, Sacrum/surgery, Chordoma, Physical therapy modalities, Cryotherapy/methods, Cryosurgery/methods

\section{Introdução}

Tumores ósseos malignos consistem aproximadamente $7 \%$ das neoplasias primárias em indivíduos menores de 20 anos de idade. Dentre todos esses, a maior prevalência é do Osteossarcoma, ocorrendo em 8,7 casos/milhão e seguido pelo Sarcoma de Ewing com 2,9 casos/milhão. Nos Estados Unidos de acordo com a American Cancer Society, a cada ano ocorrem aproximadamente 1.300 .000 casos novos de câncer ${ }^{(1-4)}$.

Obtendo uma incidência anual entre 0,1 e 0,8 a cada 1.000,000 de caos o cordoma é um tumor maligno primário de baixo grau que tem origem mesodérmica da notocorda. Acomete mais indivíduos na sexta década de vida tendo a coluna vertebral a sua localidade mais frequente. $\mathrm{O}$ cordoma aparece de forma mais comumente na região sacrococcígea $50 \%$ dos casos, podendo também ser localizado nas regiões móveis da coluna e esfeno-occipital ${ }^{(2,5-7)}$.

O cordoma é considerado um tumor resistente à radioterapia, para a sua realização é necessário dosagens superiores a $60 \mathrm{~Gy}$, mas, devido à proximidade das estruturas nervosas existe a possibilidade de serem danificadas. O uso da quimioterapia ainda não tem provado ser útil, sendo assim a ressecção em bloco cirúrgico é o tratamento primário para este tumor ${ }^{(8)}$.

Marcove et al em $1978^{(9)}$ foram os pioneiros a utilizar a crioterapia no tratamento do tumor de células gigantes. Neste estudo os autores descreveram a sua técnica através do derramamento de nitrogênio líquido com temperatura entre $-80^{\circ}$ ou $-120^{\circ} \mathrm{C}$ para poder promover o congelamento das paredes ósseas já curetadas. A crioablação induz a necrose do tecido por meio do resfriamento rápido, onde ocorrerá a formação de gelo intra e extracelular com a temperatura abaixo de zero grau. De acordo extensão e ou agressão da lesão tumoral a terapia pode variar de 5 a $10 \mathrm{mi}-$ nutos, esta é dividida em ciclos de 2 a 3 minutos de aplicação do criocongelamento seguida de um rápido descongelamento ${ }^{(9)}$.

Atualmente, o foco do tratamento mudou, ou seja, a preocupação passou a ser também a qualidade de vida que ele vai ter durante e após o tratamento oncológico. A fisioterapia oncológica é um dos procedi- mentos que estão sendo adotados nesse sentido, tanto após uma cirurgia de câncer como também durante todo o tratamento. Esse recurso pode ser utilizado em todos os casos, como nos de câncer de mama, tumores de cabeça e pescoço, além dos cânceres relacionados ao sistema músculo-esquelético ${ }^{(7,10-12)}$.

O objetivo da presente pesquisa foi descrever o efeito da intervenção fisioterapêutica na funcionalidade do paciente no pós-operatório de sacrectomia parcial associado ao criocongelamento. Para divulgação das informações do relato é importante mostrar que o paciente consentiu a publicação dos dados assinando um termo de consentimento livre e esclarecido e o trabalho foi aprovado pelo Comitê de Ética da Irmandade da Santa Casa de Misericórdia de São Paulo.

\section{Apresentação do Caso}

Paciente L.N.C 26 anos de idade, cor de pele branca, estado civil solteira, natural da cidade de Salvador-BA previamente hígida apresentou quadro álgico na região lombar há 2 anos, principalmente enquanto praticava atividades físicas. A paciente procurou serviço médico na cidade de Salvador e foi solicitado no dia 09/06/10 um exame de Ressonância Magnética, esta evidenciou uma imagem sugestiva de neoplasia de aproximadamente $5 \mathrm{~cm}$ anterior ao sacro, o serviço médico no qual realizou atendimento encaminhou a paciente para Irmandade da Santa Casa de Misericórdia de São Paulo. No dia 30/04/2011 foi internada na IMSC-SP e avaliada pelo grupo da Oncologia Ortopédica desta instituição, no qual foi solicitado uma primeira biópsia do material retirado da região sacral e diagnosticado um tumor maligno (Cordoma). Após discussão do caso entre a equipe médica do grupo do Tumor foi decidido realizar ressecção tumoral através da sacrectomia parcial associado à aplicação da técnica de Criocongelamento para eliminar as células cancerígenas. O procedimento cirúrgico foi realizado pela via de acesso posterior no dia 17/05/2011, a paciente evoluiu no pós-operatório com déficit de sensibilidade, fraqueza da musculatura do assoalho pélvico e perda total do movimento de dorsiflexão do tornozelo esquerdo sendo necessário uma órtese para manter o tornozelo em neutro prevenindo assim contratura/deformidade e facilitar a marcha. Recebeu acompanhamento da equipe de fisioterapia musculoesquelética da IMSCM-SP que tinha os seguintes objetivos; Prevenir complicações; Prevenir deformidades; Melhorar quadro álgico; Melhorar a sensibilidade dos MMIIS; Melhorar a amplitude de movimento; Melhorar a contratilidade muscular; Promover funcionalidade. O tratamento fisioterapêutico com a paciente consistiu: desensibilização dos MMIIS com diferentes texturas: esponjas, escovas, 
pincel e o gelo trabalhando a sensibilidade térmica; Cinesioterapia com exercícios ativo-livre para o MIDe exercício passivo para MIE dos movimentos de flexão / extensão do joelho; dorsiflexão/flexão plantar /inversão/eversão, dos tornozelos todos realizados 3x15; Estabilização segmentar (CORE) a conscientização e contração da musculatura profunda (Transverso do abdômen) com a periférica associando aos movimentos respiratórios e posteriormente evoluindo com a movimentação dos MMSS/MMIIS(12-13). Eletroterapia para promover analgesia com Corrente Interferencial Vetorial (CIV) e a Eletroestimulação muscular através da corrente Russa com os seguintes parâmetros: $20 \%$ ciclo de trabalho, frequência de $50 \mathrm{~Hz}, \mathrm{~T}-\mathrm{ON}$; $10 \mathrm{seg}$ e T-OFF; 20seg, tempo de aplicação de 10 Min. e de acordo com a evolução associando ao exercício isométrico e resistido com caneleiras ou Theraband (faixa elástica) para quadríceps, ísquiostibiais, dorsiflexores e flexores plantares; Tapping de deslizamento associado a movimentação ativo-assistida $3 \times 15$; treino de marcha com a descarga de peso iniciada a partir da 12 semana de pós-operatório devido a uma deiscência na região do acesso cirúrgico, retardando o tratamento fisioterapêutico. De acordo com a evolução foi adicionado na terapia à resistência com pesos, elásticos e aumentado o grau de dificuldade no treino de equilíbrio em solos instáveis. A fisioterapia foi realizada numa frequência de 3 vezes por semana.

No primeiro contato com a equipe de fisioterapia e após um ano de tratamento foi realizada uma avaliação funcional através do Escore Musculoskeletal Tumor Society (MSTS) e o Escore Toronto Extremity Salvage Score - TESS com o objetivo de mensurar a evolução funcional da paciente. Os dados referentes a o Escore Musculoskeletal Tumor Society (MSTS) Pré e pós-tratamento estão ilustrados na Tabela 1 ). Foram utilizados valores de 0 a 5, assim distribuídos: (5- Excelente/ 4-3 Bom/ 2-1 Regular/ 0- Ruim). Após a análise de cada item, foi realizada a classificação categórica do resultado funcional global, onde esta resume o resultado apresentado pela MSTS. Antes do tratamento a paciente apresentou um resultado

Tabela 1

\begin{tabular}{|c|c|c|}
\hline \multicolumn{3}{|c|}{ Tabela 1} \\
\hline MSTS & Pré-tratamento & Pós-tratamento \\
\hline Dor & Ruim & Excelente \\
\hline Função & Ruim & Bom \\
\hline Emocional & Ruim & Bom \\
\hline Suporte & Ruim & Bom \\
\hline Marcha & Ruim & Bom \\
\hline Deformidade & Ruim & Regular \\
\hline
\end{tabular}

Ruim e após um ano de tratamento fisioterapêutico a paciente apresentou um escore Bom. De acordo com o escore TESS a pontuação de cada questão varia de 0 a 5 pontos, nossa paciente apresentou um escore total de $28 \%$ no pré-tratamento e evoluiu para $48 \%$ após um ano de acompanhamento com Grupo do Tumor e a Equipe de Fisioterapia da Irmandade Santa Casa de Misericórdia (IMSCSP) $)^{(10-11,14-15)}$.

\section{Discussão}

Nos Estados Unidos menos de um a cada 1 milhão de pessoas é a incidência do cordoma nas pessoas com câncer ${ }^{(11)}$. As possibilidades de tratamento estão limitadas nesta lesão tumoral por não responder bem a radioterapia e a quimioterapia ${ }^{(12-13)}$, sendo assim a ressecção cirúrgica o tratamento primário desta lesão ${ }^{(14)}$. A via de acesso cirúrgico é a primeira escolha nesta abordagem terapêutica, a via de acesso radical (anterior) raramente é realizada devido à localidade do tumor e sua relação anatômica com os tecidos e órgãos vizinhos. Devido a este motivo a via de acesso cirúrgico da nossa paciente foi posterior ${ }^{(15-16)}$.

Yonemoto et al (1999) ${ }^{(17)}$ relatam que existe a possibilidade do cordoma penetrar ou infiltrar nos músculos glúteos, por isso é importante a avaliação com ressonância magnética para prevenção de uma recidiva. Caso isto aconteça a via de acesso posterior permite uma boa familiarização com anatomia local o que facilita a ressecção da musculatura glútea ou piriforme para ressecar totalmente a lesão(17).

Estudos nos mostram que a ressecção em bloco ocorre em até $50 \%$ dos pacientes, mesmo tratado de forma agressiva o cordoma de sacro vem apresentando um prognóstico melhor em comparação com relatos anteriores onde as taxas de sobrevida de cinco anos têm variado de $73 \%$ a $86 \%$, enquanto que a taxas de sobrevivência anual têm aumentado de $49 \%$ a $71 \%$. Yang et al (2010) ${ }^{(19)}$ realizaram um estudo com 30 pacientes com cordoma sacral que foram submetidos à embolização pré-operatória utilizando Gelfoam como agente embólico. A média de perda sanguínea foi de $1200 \mathrm{ml}$ e o valor médio no pós-operatório de transfusões foi $1080 \mathrm{ml}$. Este estudo mostrou que a embolização pré-operatória de cordomas sacrais parece ser eficaz na remoção máxima da lesão e uma menor perda sanguínea intra-operatório ${ }^{(18-19)}$.

Bergh et al $(2000)^{(20)}$ realizaram uma série de casos com 39 pacientes, onde 30 eram cordoma de sacro e os outros nove na extensão da coluna móvel, o tempo médio de acompanhamento foi de 8,1 anos variando de 5 a 20, todos realizaram a ressecção da lesão por cirurgia. Nos seus resultados 17 pacientes (44\%) apresentaram recidivas locais e 11 apresentaram metástases $(28 \%)$ e no que diz respeito à taxa de sobrevida foi de 
$84 \%$ em 5 anos, $64 \%$ em 10 anos, $52 \%$ em 15 anos e $52 \%$ em 20 anos, neste estudo a recorrência local teve associação estatística com o aumento do risco de metástase e morte relacionada ao tumor ${ }^{(20)}$.

Stacchiotti et al(2010) ${ }^{(21)}$ realizaram um estudo retrospectivo a partir de 2009 com 138 pacientes, destes $78 \%$ (sacro) e o restante presente na coluna móvel. Todos os pacientes com uma média de acompanhamento de 142 meses onde foram submetidos à cirurgia de ressecção para remover a lesão tumoral. Nos seus resultados a sobrevivência destes pacientes foi de 5 anos em $78 \%$ dos casos e 10 anos em $26 \%$, mas a maioria destes indivíduos foram a óbito devido à lesão tumoral primária ou com a presença de metástase ${ }^{(21)}$.

O tratamento fisioterapêutico se mostrou eficaz no resultado funcional da paciente mostrando o benefício alcançado com a intervenção, deixando uma perspectiva de pesquisas futuras com um número maior de pacientes e comparando diferentes abordagens de tratamento.

\section{Conclusão}

O acompanhamento multidisciplinar do grupo da Oncologia Ortopédica junto à equipe de fisioterapia da Irmandade Santa de Misericórdia de São Paulo garantiu uma melhora no estado funcional da paciente de acordo com escore Score Musculoskeletal Tumor Society (MSTS) e o Toronto Extremity Salvage Score (TESS).

\section{Referências}

1. Marina N, Gebhardt M, Teot L, Gorlick R. Biology and therapeutic advances for pediatric osteosarcoma. Oncologist. 2004; 9(4):422-41.

2. Sciubba DM, Cheng JJ, Petteys RJ, Weber KL, Frassica DA, Gokaslan ZL. Chordoma of the sacrum and vertebral bodies. J Am Acad Orthop Surg. 2009; 17(11):708-17.

3. Próspero JD. Tumores ósseos. São Paulo: Roca; 2001. 280p.

4. Camargo OP. Conduta atual nas lesões ósseas metastáticas. Rev Bras Ortop. 2004; 39(6):273-82.

5. Oliveira OLR, Carneiro PCA, Sales Filho R, Oliveira DP. Câncer de mama em mulheres jovens: aspectos epidemiológicos. Rev Soc Bras Cancerol. 1999; 36(2):6-9.

6. Ferrari BL, Marinho RM, Rocha MLL, Silva HMS. Considerações sobre as doenças da mama. In: Baracho E. Fisioterapia aplicada à obstetrícia: aspectos de ginecologia e neonatologia. $3^{\mathrm{a}}$. ed. Rio de Janeiro: Medsi; 2002. p. 422-47.

7. Healey JH, Lane JM. Chordoma: a critical review of diagnosis and treatment. Orthop Clin North Am. 1989; 20(3):417-26.
8. Marcove RC, Sheth DS, Brien EW, Huvos AG, Healey JH. Conservative surgery for giant cell tumors of the sacrum. The role of cryosurgery as a supplement to curettage and partial excision. Cancer. 1994; 74(4):1253-60.

9. Marcove RC, Weis LD, Vaghaiwalla MR, Vaghaiwalla MR, Pearson R, Huvos AG. Cryosurgery in the treatment of giant cell tumor of bone. A report of 52 consecutive cases. Cancer. 1978; 41(3):957-69.

10. Enneking WF, Dunham W, Gebhardt MC, Malawar M, Pritchard MD. A system for the functional evaluation of reconstructive procedures after surgical treatment of tumors of the musculoskeletal system. Clin Orthop Rel Res. 1993; (286):241-6.

11. Davis AM, Wright JG, Williams JI, Bombardier C, Griffin A, Bell RS. Development of measure of physical function for patients with bone and soft tissue sarcoma. Qual Life Res. 1996; 5(5):50816.

12. Fasig DH, Dupont WD, LaFleur BJ, Cates JM, Olson SJ. Immunohistochemical analysis of receptor tyrosine kinase signal transduction activity in chordoma. Neuropathol Appl Neurobiol. 2008; 34(1):95-104.

13. Panjabi MM. The stabilizing system of the spine, part I: function, dysfunction, adaption and enhancement. J Spinal Disord. 1992; 5(4):383-9.

14. McMaster ML, Goldstein AM, Bromley CM, Ishibe N, Parry DM. Chordoma: incidence and survival patterns in the United States, 1973-1995. Cancer Causes Control 2001; 12(1):1-11.

15. Gennari L, Azzarelli A, Quagliuolo V. A posterior approach for the excision of sacral chordoma. J Bone Joint Surg Br. 1987; 69(4):565-8.

16. Cheng EY, Ozerdemoglu RA, Transfeldt EE, Thompson RC Jr. Lumbosacral chordoma. Prognostic factors and treatment. Spine (Phila Pa 1976). 1999; 24:1639-45.

17. Yonemoto T, Tatezaki S, Takenouchi T, Ishii T, Satoh T, Moriya H. The surgical management of sacrococcygeal chordoma. Cancer. 1999; 85(4):878-83.

18. Samson IR, Springfield DS, Suit HD, Mankin HJ. Operative treatment of sacrococcygeal chordoma. A review of twenty-one cases. J Bone Joint Surg Am 1993; 75(10):1476-84.

19. Yang H, Zhu L, Ebraheim NA, Liu J, Shapiro A, Castillo S, et al. Surgical treatment of sacral chordomas combined with transcatheter arterial embolization. J Spinal Disord Tech .2010; 23(1):47-52.

20. Bergh P, Kindblom LG, Gunterberg B, Remotti F, Ryd W, MeisKindblom JM. Prognostic factors in chordoma of the sacrum and mobile spine: a study of 39 patients. Cancer. 2000; 88(9):2122-34.

21. Stacchiotti S, Casali PG, Lo Vullo S, Mariani L, Palassini E, Mercuri $\mathrm{M}$, et al. Chordoma of the mobile spine and sacrum: a retrospective analysis of a series of patients surgically treated at two referral centers. Ann Surg Oncol. 2010; 17(1):211-9.

Trabalho recebido: 30/01/2019

Trabalho aprovado: 19/06/2019

Trabalho publicado: 26/06/2019 